\title{
Diversity of Functional Astroglial Properties in the Respiratory Network
}

\author{
Dennis Graß, ${ }^{1}$ Petra G. Pawlowski, ${ }^{2 \star}$ Johannes Hirrlinger ${ }^{2,3 *}$ Nestoras Papadopoulos, ${ }^{1}$ Diethelm W. Richter, ${ }^{1}$ \\ Frank Kirchhoff, ${ }^{2}$ and Swen Hülsmann ${ }^{1}$ \\ ${ }^{1}$ Department of Neuro- and Sensory Physiology, Center for Physiology and Pathophysiology, Georg-August-University, 37073 Göttingen, Germany, \\ ${ }^{2}$ Department of Neurogenetics, Max Planck Institute of Experimental Medicine, 37075 Göttingen, Germany, and ${ }^{3}$ DFG-Research Center for Molecular \\ Physiology of the Brain, 37073 Göttingen, Germany
}

A population of neurons in the caudal medulla generates the rhythmic activity underlying breathing movements. Although this neuronal network has attracted great attention for studying neuronal aspects of synaptic transmission, functions of glial cells supporting this neuronal activity remain unclear. To investigate the role of astrocytes in the respiratory network, we applied electrophysiological and immunohistochemical techniques to characterize astrocytes in regions involved in the generation and transmission of rhythmic activity. In the ventral respiratory group and the hypoglossal nucleus (XII) of acutely isolated brainstem slices, we analyzed fluorescently labeled astrocytes obtained from TgN(GFAP-EGFP) transgenic mice with the whole-cell voltage-clamp technique. Three subpopulations of astrocytes could be discerned by their distinct membrane current profiles. A first group of astrocytes was characterized by nonrectifying, symmetrical and voltage-independent potassium currents and a robust glutamate transporter response to D-aspartate. A second group of astrocytes showed additional A-type potassium currents, whereas a third group, identified by immunolabeling for the glial progenitor marker NG2, expressed outwardly rectifying potassium currents, smaller potassium inward currents, and only minimal D-aspartateinduced transporter currents. Astrocytes of all groups showed kainate-induced inward currents.

We conclude that most of the astrocytes serve as a buffer system of excess extracellular glutamate and potassium; however, a distinct cell population (NG2-positive, A-type potassium currents) may play an important role for network plasticity.

Key words: brainstem; GFAP (glial fibrillary acidic protein); EGFP; glutamate; potassium (K); neuron-glia interaction

\section{Introduction}

Neurons in the caudal medulla participate in the generation of the rhythmic activity of breathing (Smith et al., 1991; Richter and Spyer, 2001; Del Negro et al., 2002). The neuronal activity of this network is transmitted synaptically to respiratory motor outputs that include cranial motoneurons, thereby controlling breathing movements. A striking feature of the brainstem respiratory network, localized within the pre-Bötzinger complex, are rhythmic bursts of action potentials leading to repetitive increases of potassium ions in the extracellular space in vitro and in vivo (Richter et al., 1978; Brockhaus et al., 1993). In addition, rhythmic motor activity depends on synaptic interactions within the network involving phasic release of neurotransmitters that include glutamate (Greer et al., 1991; Koshiya and Smith, 1999; Richter and Spyer, 2001). It follows that extracellular potassium (Rybak et al., 2003) and glutamate will control neuronal bursting by setting

\footnotetext{
Received Sept. 1, 2003; revised Nov. 28, 2003; accepted Dec. 9, 2003.

This work was supported by The Deutsche Forschungsgemeinschaft through the DFG-Research Center for Molecular Physiology of the Brain. We thank M. Müller. C. Neusch, J. Paton, and W. Zhang for comments on this manuscript, M. Quintela-Schneider for help with histology, and A.-A. Grützner and C. Bartje for technical assistance. *P.G.P. and J.H. contributed equally to this study.

Correspondence should be addressed to Dr. Swen Hülsmann, Abteilung Neuro- und Sinnesphysiologie Zentrum Physiologie und Pathophysiologie, Georg-August-Universität, Humboldtallee 23, D-37073 Göttingen, Germany. E-mail: sh@ukps.gwdg.de or shuelsm2@uni-goettingen.de.

DOI:10.1523/JNEUROSCI.4022-03.2004

Copyright $\odot 2004$ Society for Neuroscience $\quad 0270-6474 / 04 / 241358-08 \$ 15.00 / 0$
}

and modulating the membrane potential. Indeed, abundant expression of different ionotropic and metabotropic glutamate receptors has been described for the brainstem (Robinson and Ellenberger, 1997; Dong and Feldman, 1999).

Astrocytes are predestined to maintain the homeostasis of the extracellular space as an efficient sink for potassium and by preventing spillover of neurotransmitters such as glutamate (Kullmann and Asztely, 1998; Rusakov and Kullmann, 1998; Hülsmann et al., 2000a). Neuronal network activity also depends on metabolic support from neighboring astrocytes (Hülsmann et al., $2000 \mathrm{~b}$ ); however, many aspects of the role of astrocytes in the vital respiratory regions of the medulla are still unknown. In the present study, we performed a systematic analysis of astrocytes in respiratory-related regions of the medulla, including the ventral respiratory group (VRG), with the pre-Bötzinger complex, and the hypoglossal motor nucleus. Identification of astrocytes was performed by GFAP promoter-controlled enhanced green fluorescent protein (EGFP) labeling of astrocytes in transgenic mice (Nolte et al., 2001). In particular, we focused our interest on those glial properties that modulate synaptic transmission, i.e., their membrane potassium conductances and their responsiveness to glutamate. Specifically, our study addressed the question of whether astrocytes in respiratory-related regions of the brainstem fulfill the requirements of potassium buffering and regulation of the extracellular glutamate concentration. 


\section{Materials and Methods}

Electrophysiology. Acute brainstem slices from TgN(GFAP-EGFP) mice from postnatal day $0-11$ were prepared as described previously (Hülsmann et al., 2000a; Nolte et al., 2001). Briefly, animals were decapitated, and the brainstem was prepared and placed in ice-cooled, carbogensaturated $\left(95 \% \mathrm{O}_{2}, 5 \% \mathrm{CO}_{2}\right)$ artificial CSF (ACSF) containing (in mM): $118 \mathrm{NaCl}, 3 \mathrm{KCl}, 1.5 \mathrm{CaCl}_{2}, 1 \mathrm{MgCl}_{2}, 1 \mathrm{NaH}_{2} \mathrm{PO}_{4}, 25 \mathrm{NaHCO}_{3}$, and 30 D-glucose; $330 \mathrm{mOsm} / 1, \mathrm{pH}$ 7.4. The brainstem was glued to an agar block with cyanacrylate glue (Loctite Deutschland GmbH, Munich, Germany), and transverse slices $(200 \mu \mathrm{m})$ were cut from the caudal medulla at the level of the pre-Bötzinger complex with a vibroslicer (Campden Instruments, Loughborough, UK). Slices were stored in ACSF at room temperature $\left(20-23^{\circ} \mathrm{C}\right)$ for at least $30 \mathrm{~min}$ before experiments were started. Subsequently, slices were transferred to the recording chamber and kept submerged by nylon fibers (Edwards et al., 1989) for mechanical stabilization. The chamber was mounted on an upright microscope (Axioscope FS, Zeiss, Oberkochen, Germany) and perfused continuously with ACSF $\left(20-23^{\circ} \mathrm{C}\right)$ at a flow rate of $5-10 \mathrm{ml} / \mathrm{min}$. Astrocytes were identified by their green fluorescence in the epifluorescence illumination (excitation $488 \mathrm{~nm}$; dichroic mirror $495 \mathrm{~nm}$; Polychrome II, TILL Photonics, Gräfelfing, Germany). Images of astrocytes were taken with a CCD camera (MicroMax, Princeton Instruments, Trenton, NJ), and the Imaging software was from Axon (Imaging workbench 2.0, Axon Instruments, Inc., Foster City, CA). Whole-cell voltage-clamp recordings were obtained with a discontinuous single-electrode voltage-clamp amplifier (NPI SEC-05L; Tamm, Munich, Germany) at switching frequencies of $40-50 \mathrm{kHz}$ (duty cycle, $25 \%$ ) or with a Multiclamp 700A amplifier (Axon Instruments). Patch electrodes were pulled from borosilicate glass capillaries (Biomedical Instruments, Zülpich, Germany) on a horizontal pipette puller (Zeitz-Instrumente, Munich, Germany) and filled with (in mM): $125 \mathrm{KCl}, 1 \mathrm{CaCl}_{2}, 2 \mathrm{MgCl}_{2}, 4 \mathrm{Na}_{2} \mathrm{ATP}, 10$ EGTA, 10 HEPES (pH adjusted to 7.2 with $\mathrm{KOH}$ ). Their resistance was 2-6 $\mathrm{M} \Omega$.

Currents were low-pass filtered at $3 \mathrm{kHz}$, sampled at $10 \mathrm{kHz}$, and recorded with "Pulse" software (v.8.53; HEKA, Lambrecht, Germany), Axograph 4.6 software, or Clampex 8.2 software (Axon Instruments) and stored for off-line analysis. Astrocytes were voltage clamped to $-80 \mathrm{mV}$ and exposed to different voltage-step protocols (see Figs. 2, 3).

For local pressure application, kainate (to activate AMPA-type glutamate receptors without desensitization) or glutamate was dissolved in HEPES-buffered ACSF containing (in mM): $118 \mathrm{NaCl}, 3 \mathrm{KCl}, 1.5 \mathrm{CaCl}_{2}$, $1 \mathrm{MgCl}_{2}, 25$ HEPES, 30 glucose, $\mathrm{pH} 7.4$, put into a glass pipette with a tip diameter of $2 \mu \mathrm{m}$, and pressure ejected $(0.2 \mathrm{bar}, 0.2-0.5 \mathrm{sec})$ onto the cells at a distance of $\sim 30-50 \mu \mathrm{m}$. D-aspartate was bath applied for 20 sec. Indirect neuronal effects induced by local kainate application were minimized by adding $0.5 \mu \mathrm{M}$ TTX and $0.1 \mathrm{mM} \mathrm{CdCl}_{2}$ to block voltage-gated sodium and calcium channels, respectively. During D-aspartate application, $50 \mu \mathrm{M}$ 6-cyano-7-nitro-quinoxaline-2,3dione (CNQX; to block AMPA-type glutamate receptors), $0.5 \mu \mathrm{M}$ TTX, and $0.1 \mathrm{~mm} \mathrm{CdCl}_{2}$ were added to ACSF. Local glutamate application was performed under blockade of synaptic transmission by 0.5 $\mu \mathrm{M}$ TTX, $0.1 \mathrm{mM} \mathrm{CdCl}_{2}, 50 \mu \mathrm{M} \mathrm{DL}$-2-amino-5-phosphopentanoic acid (AP5; block of NMDA-type glutamate receptors), $20 \mu \mathrm{M}$ bicuculline, and $10 \mu \mathrm{M}$ strychnine (block of the inhibitory $\mathrm{GABA}_{\mathrm{A}}$ and glycine receptors, respectively).

Data were analyzed with IGOR Pro (WaveMetrics) and expressed as mean \pm SEM (SE). Student's $t$ tests were used to determine statistical significance (Sigma Plot software, SPSS Inc., Chicago, IL). Results were considered significant if $p<0.05$.

Immunohistochemistry and morphological analysis. TgN(GFAP-EGFP) mice from postnatal day 2-23 were anesthetized with $2.5 \%$ 2,2,2tribromoethanol in $\mathrm{H}_{2} \mathrm{O}(100 \mu \mathrm{l} / 10 \mathrm{gm})$ and perfused transcardially with HBSS followed by perfusion with $4 \%$ paraformaldehyde in $100 \mathrm{~mm}$ phosphate buffer for $15 \mathrm{~min}$. The brainstem was removed and incubated in $4 \%$ paraformaldehyde in $100 \mathrm{~mm}$ phosphate buffer for $2-6 \mathrm{hr}$ at $4^{\circ} \mathrm{C}$. A series of transversal sections $(40-60 \mu \mathrm{m})$ were cut from the caudal medulla with a Vibratome (Leica VT 1000S, Leica Instruments, Nussloch, Germany). Immunohistochemical labeling was performed on freefloating slices $(60 \mu \mathrm{m})$ at room temperature. Cells were permeabilized with $0.4 \%$ Triton X-100 in PBS for 30 min and then blocked for $30 \mathrm{~min}$ in $10 \%$ fetal calf serum (FCS) with $0.2 \%$ Triton X-100 in PBS. The slices were incubated overnight at $4^{\circ} \mathrm{C}$ in $2 \%$ FCS and $0.05,0.1$, or $0.5 \%$ Triton $\mathrm{X}-100$ in PBS with one or in case of double-stainings two of the following first antibodies: polyclonal antibody against glutamate-aspartate transporter (GLAST) (guinea pig, 1:2000), glial glutamate transporter (GLT-1) (guinea pig, 1:2000), or monoclonal antibody against NG2 (rat, 1:100), TUC-4 [ToAD (turned on after division)/Ulip/CRMP] (rabbit, 1:2500), and GFAP (rabbit, 1:250). Slices were washed twice in PBS for 5 min and incubated with secondary, Cy3-conjugated as well as Cy5conjugated antibodies (1:1000) for $2 \mathrm{hr}$. After washing in PBS, slices were mounted on object slides with aqua-polymount (Polysciences, Warrington, PA) or Immu-Mount (Shandon, Pittsburgh, PA). First antibodies were obtained from Chemicon International (Temecula, CA), except the GFAP antibody, which was purchased from Dako Cytomation (Glostrup, Denmark), and the NG2-antibody, which was a gift from Dr. J. Trotter (Department for Molecular Cell Biology, University of Mainz, Mainz, Germany) (Diers-Fenger et al., 2001). Secondary antibodies for GLAST and GLT-1 stainings were obtained from Chemicon; those used for double-staining experiments were obtained from Dianova (Hamburg, Germany). Immunostainings were visualized using a confocal laser-scanning microscope (LSM 510, Axiovert 200, Zeiss, Oberkochen, Germany). EGFP was excited at $488 \mathrm{~nm}$ and detected through a $500-550 \mathrm{~nm}$ bandpass filter. Cy 3 was excited at $543 \mathrm{~nm}$ and detected with a $565-615 \mathrm{~nm}$ bandpass filter. Cy5 was exited at $633 \mathrm{~nm}$ and detected with a $650 \mathrm{~nm}$ longpass filter. All confocal images were stored and processed with the Zeiss LSM Software. NG2-positive cells in the ventral respiratory group were counted in $2-\mu \mathrm{m}$-thick $\mathrm{z}$-stacks; counting of EGFP-expressing cells was done in $50-\mu \mathrm{m}$-thick z-stack images obtained by two-photon laser microscopy (Axoscope FS2mot, LSM510 NLO, Zeiss) using a 5 W Verdi pump laser and a Mira titaniumsapphire infrared laser (Coherent, Dieburg, Germany). Two investigators, advised to assign single cells into either group, performed the evaluation of fluorescence intensity: bright cells versus weakly fluorescent cells.

During electrophysiological experiments, selected cells were filled with the fluorescent dye Alexa Fluor 568 (Molecular Probes, Eugene, OR) for 5-10 min. After filling, slices were fixed in $4 \%$ formaldehyde for $2 \mathrm{hr}$ and visualized by confocal microscopy as described above or by CCD camera.

\section{Results}

To assess the function of astrocytes in respiratory-related regions of the brainstem and their potential in regulating synaptic transmission and rhythmic neuronal activity, we performed an electrophysiological study on astrocytes of the ventral respiratory group and the hypoglossal nucleus.

\section{Appearance of astrocytes in the respiratory network}

Identification of astrocytes was unequivocal in brain slice preparations from the $\mathrm{TgN}$ (GFAP-EGFP) mouse line (Nolte et al., 2001). Interestingly, the level of EGFP expression as indicated by fluorescence intensity varied among astrocytes of both the ventral respiratory group (Fig. $1 A, \mathrm{VRG}$ ) and the hypoglossal motor nucleus (Fig. $1 B$ ). In principle, two astroglial populations could be distinguished as being either brightly or weakly fluorescent. To quantify the distribution of differently labeled astrocytes, we counted the number of each group within three-dimensional stacks of images of a volume of $150 \times 150 \times 50 \mu \mathrm{m}(n=4)$ recorded by two-photon laser scanning microscopy. This revealed a balanced distribution of highly $(46.7 \pm 4.0 \%)$ and weakly (53.3 $\pm 4.0 \%)$ EGFP-expressing cells. Morphologically, astrocytes with high fluorescence intensity were characterized by round or multipolar somata with numerous highly ramified processes (Fig. 1A), whereas the identification of processes in cells with low EGFP expression was more difficult. For this purpose, several cells with weak $(n=8)$ (Fig. $1 C-E)$ and high $(n=11)$ (Fig. 
$1 G-I)$ fluorescence intensity were filled with the red fluorescent dye Alexa Fluor 568 via the patch pipette. With this procedure, process extensions of $>20 \mu \mathrm{m}$ in each direction in all cell types were revealed by laser scanning microscopy (Fig. 1) and CCD camera images (Fig. 2). Although the extension of processes was similar in both cell types, the branching of processes was higher in cells with high EGFP expression.

\section{Electrophysiological analysis of astrocytes}

To analyze for differences in whole-cell membrane currents, EGFP-labeled astrocytes were voltage clamped to potentials ranging from -150 to $70 \mathrm{mV}$ by changing the voltage stepwise in $10 \mathrm{mV}$ increments, whereas corresponding currents were recorded to determine current-voltage $(I-V)$ relations (Fig. 2). Three populations of astrocytes could be distinguished, according to their potassium currents.

(1) Astrocytes with almost symmetrical, inwardly and outwardly directed potassium currents resulting in a linear $I-V$ curve (for both the initial part and the steady state of the current response) were designated as passive $(n=85)$ (Fig. $2 A-$ $D)$. The resting membrane potential of these astrocytes was $-84 \pm 0.9 \mathrm{mV}$ (Fig. $2 \mathrm{M})$. Consistently, the input resistance indicative for high resting potassium conductances around the resting membrane potential was $31 \pm 3 \mathrm{M} \Omega($ Fig. $2 N$ ). The amplitude of the noninactivating inward current resulting from a voltage step to $-150 \mathrm{mV}$ was $-2.8 \pm 0.2 \mathrm{nA}$.

(2) Astrocytes that showed an initial transient outward current during voltage steps to potentials that were more positive than $-50 \mathrm{mV}$ and a linear $I-V$ curve during the steady state $(n=64)$ (Fig. $2 E-H)$ were designated as intermediate astrocytes. These cells had an average resting membrane potential of $-84 \pm 1.1 \mathrm{mV}$. The input resistance $(33 \pm 3$ $\mathrm{M} \Omega$ ) and the inward current at $-150 \mathrm{mV}(-2.6 \pm 0.2 \mathrm{nA})$ were comparable with passive astrocytes.

(3) A third group of cells was characterized by an outwardly rectifying $I-V$ curve and lack of inward currents. The initial current-response showed characteristics of an A-type potassium current (see below), whereas there was also a noninactivating outwardly rectifying steady-state current $(n=34)$ (Fig. $2 I-L)$. The resting membrane potential was less negative $(-66 \pm 4 \mathrm{mV})$ as compared with passive and intermediate astrocytes $(p<$ 0.001) (Fig. $2 M$ ), reflecting a significantly higher input resistance of $319 \pm 43 \mathrm{M} \Omega(p<0.001)$ (Fig. $2 N)$. The inward currents at $-150 \mathrm{mV}$ were significantly smaller than in passive astrocytes $(-0.4 \pm 0.1 \mathrm{nA} ; p<0.001)$. These cells were designated outwardly rectifying astrocytes.

Transient outward currents, especially A-type potassium currents, have been described in different preparations for astrocytes and progenitor cells of various brain regions (Jabs et al., 1994;
Stewart et al., 1999; Bordey and Sontheimer, 2000; Filippov et al., 2003). We quantified the A-type potassium currents in the different types of astrocytes by a specific prepulse protocol (Fig. 3). The transient outward current started to be activated at $-40 \mathrm{mV}$ and inactivated after 50-80 msec. The largest amplitudes of the A-Type $\mathrm{K}^{+}$current $(1.75 \pm 0.31 \mathrm{nA})$ at a membrane potential of $+70 \mathrm{mV}$ were detected in outwardly rectifying astrocytes $(n=$ 24). Application of $1 \mathrm{~mm} 4$-aminopyridine reversibly reduced the transient outward current by $70.1 \pm 7.7 \%(n=3$; data not shown). The $I-V$ curve of the remaining current was comparable with delayed rectifier potassium current. The A-type current in intermediate astrocytes was significantly smaller as compared with outwardly rectifying cells $(0.72 \pm 0.17 \mathrm{nA} ; n=46 ; p<0.01)$. The subtraction protocol revealed almost no A-type currents in passive astrocytes $(0.13 \pm 0.04 \mathrm{nA} ; n=55 ; p<0.001)$ (Fig. $3 E)$.

\section{Correlation between EGFP fluorescence and $I-V$ relation}

Identified astrocytes were compared with respect to their EGFP fluorescence and $I-V$ relations. Of 70 astrocytes with high fluorescence intensity (recorded by a CCD camera during electrophysiological characterization) (Fig. 2), 48 astrocytes (69\%) were 
A

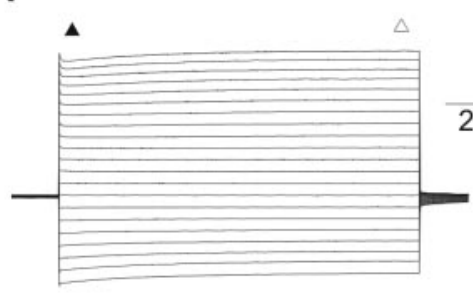

B

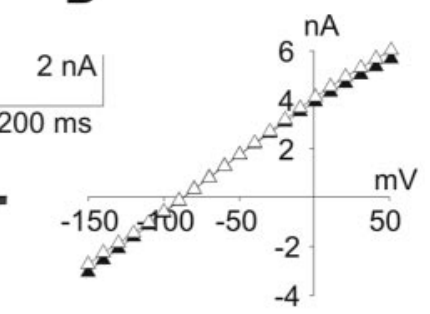

$\mathrm{mV}$
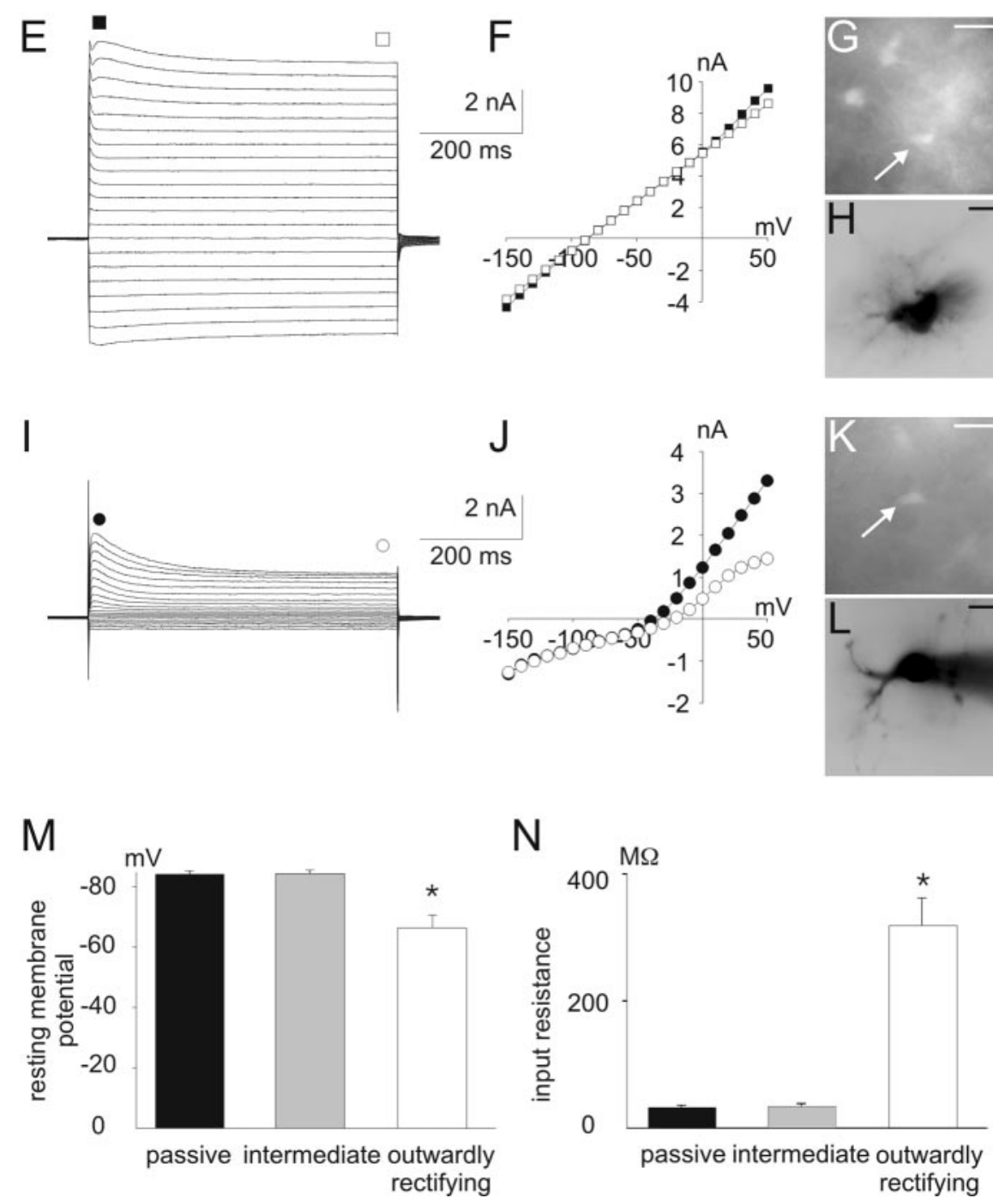

Figure 2. Different $I-V$ relations of astrocytes in the ventral respiratory group. $A-D$, Whole-cell voltage-clamp recordings $(A)$, $I-V$ curve $(B)$, and corresponding $C C D$ camera images $(C, D)$ from a passive astrocyte in the ventral respiratory group. $E-L$, Corresponding data from an intermediate cell $(E-H)$ and from an outwardly rectifying cell $(I-L)$. CCD images in $C, G$, and $K$ represent EGFP fluorescence; images in $D, H$, and $L$ represent Alexa Fluor 568 filling of the cells in $C, G$, and $K$, respectively. $A-L$, Whole-cell membrane currents were recorded by stepping the holding potential from -150 to $+50 \mathrm{mV}$ ( $10 \mathrm{mV}$ increments) starting at a holding potential of $-80 \mathrm{mV}$. For $/-V$ relations, current amplitudes at the beginning (closed symbols) and at the end of the voltage pulses (open symbols) were plotted against the holding potential. $M, N$, Resting membrane potential $(M)$ and input resistance $(N)$ in different astrocytes as distinguished in $A-L$ (data are given as mean $\pm S E ;{ }^{*} p<0.05$ ). Scale bars, $20 \mu \mathrm{m}$.

passive, $21(30 \%)$ were intermediate, and only 1 single cell had outwardly rectifying properties. As evident from data described above, high EGFP fluorescence was highly predictive for cells with a more negative resting membrane potential and a high resting potassium conductance, as typical for passive and inter-
.
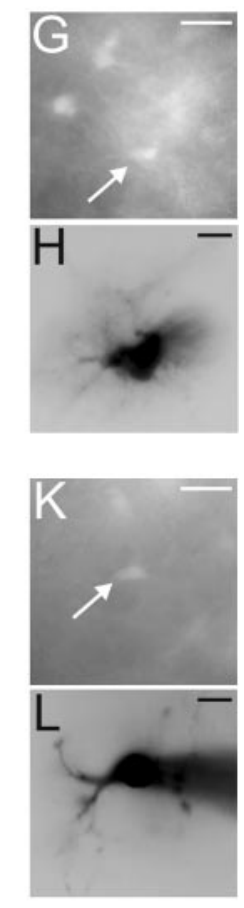

mediate cells. In contrast, low fluorescence was less predictive for the individual cell type. From 72 cells selected for their low fluorescence, 20 cells (28\%) were passive, $25(34 \%)$ were intermediate, and 27 cells (38\%) had outwardly rectifying properties. Almost all cells (one exception) with outward rectification and lack of inward currents belonged to the group of weakly fluorescent astrocytes.

\section{Astrocytes differentially express} functional glutamate transporters Because glutamate is the major excitatory transmitter of the respiratory network in the brainstem, we assessed the responsiveness of astrocytes to glutamate by analyzing the expression of functional glutamate transporters in the different astrocyte subpopulations. Astroglial removal of glutamate from the extracellular space is known to modulate synaptic transmission (Mennerick et al., 1999). Membrane currents were recorded in response to application of the glutamate transporter agonist D-aspartate. To minimize indirect effects from neighboring neurons, voltage-gated sodium and calcium channels as well as AMPA-type glutamate receptors were blocked by $500 \mathrm{nM}$ TTX, $100 \mu \mathrm{M} \mathrm{CdCl}_{2}$, and $50 \mu \mathrm{M}$ CNQX, respectively, added to the bath solution. At a holding potential of $-80 \mathrm{mV}$, application of D-aspartate (1 $\mathrm{mm}$ ) elicited inward currents (Fig. 4A-C). In passive astrocytes, $D$-aspartate evoked an inward current of $241 \pm 56 \mathrm{pA}(n=8)$ (Fig. $4 A, D$ ). There was no significant difference of the $D$-aspartate current in intermediate astrocytes $(169 \pm 28 \mathrm{pA} ; n=12)$. In contrast, outwardly rectifying astrocytes showed only small inward currents $(39 \pm 17 \mathrm{pA} ; n=11 ; p<0.001)$ (Fig. $4 C, D)$.

In addition, we detected high expression levels of glutamate transporters on passive astrocytes by immunostaining. Positive immunoreactivity for both of the glia-specific glutamate transporters, GLT-1 (Fig. 5A-C) and GLAST (Fig. 5D$F$ ), was almost evenly distributed on processes and somata of astrocytes with intensive EGFP fluorescence. In astrocytes with low fluorescence, however, expression of GLT-1 or GLAST was not detectable.

\section{Presence of AMPA-kainate currents}

Ionotropic glutamate receptors, especially AMPA-type glutamate receptors, and outwardly rectifying potassium channels are co-expressed in astrocytes of different brain regions, e.g., in young Bergmann glial cells of the cerebellum (Müller et al., 1992, 1994) and astrocytes of the hippocampus (Jabs et al., 1994; Matthias et al., 2003). Concerted action of ionotropic glutamate receptor-mediated depolarization 

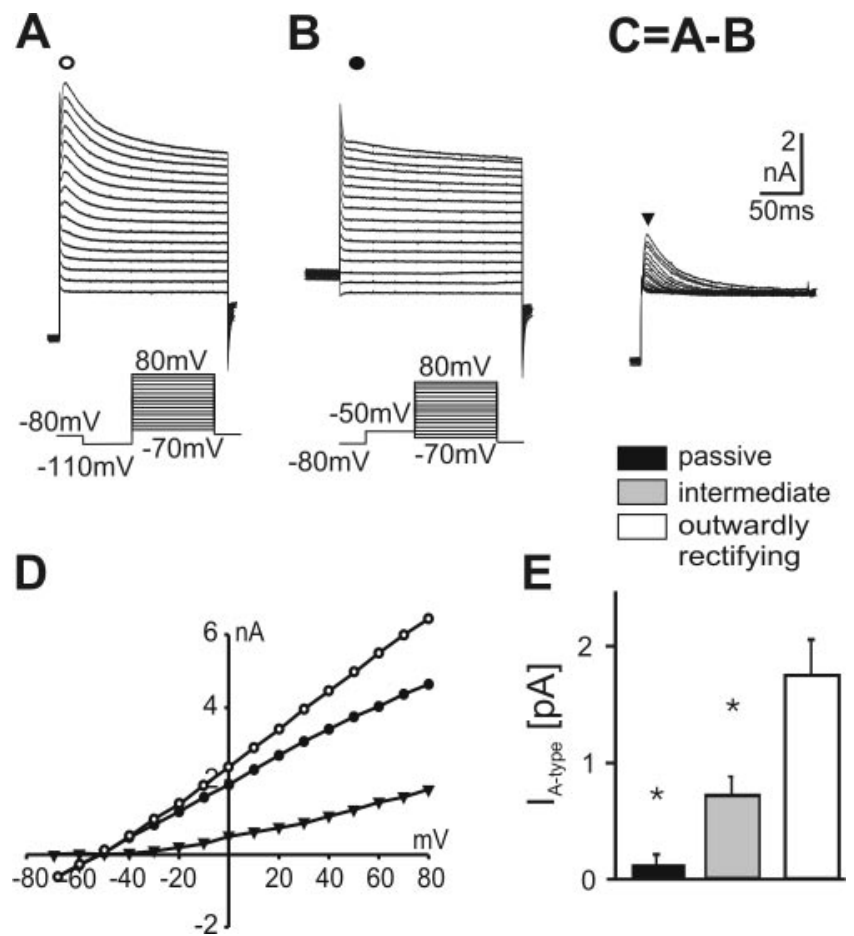

Figure 3. Expression of A-type $\mathrm{K}^{+}$currents in astrocytes of the ventral respiratory group. $A-D$, Whole-cell voltage-clamp recordings in an intermediate astrocyte from the ventral respiratory group exposed to voltage pulses from -70 to $+80 \mathrm{mV}$ for $400 \mathrm{msec}$ after prepulses to $-110 \mathrm{mV}(A$, open circles in $D)$ and $-50 \mathrm{mV}$ ( $B$, filled circles in $D)$. Point-by-point subtraction of the two recordings reveals the isolated transient current, which has the properties of an A-type $\mathrm{K}^{+}$current (O.D, Calculated A-type currents (black triangles) determined $10 \mathrm{msec}$ after the beginning of the voltage pulses are plotted against membrane potential. I- $V$ curves show an activation potential of the $\mathrm{K}^{+}$channels at $-40 \mathrm{mV}$ that is characteristic for A-type current. $E$, Amplitudes of A-type $\mathrm{K}^{+}$currents derived from subtraction protocol (at $+70 \mathrm{mV}$ ) in different types of astrocytes (data are given as mean $\pm \mathrm{SE}$ ).

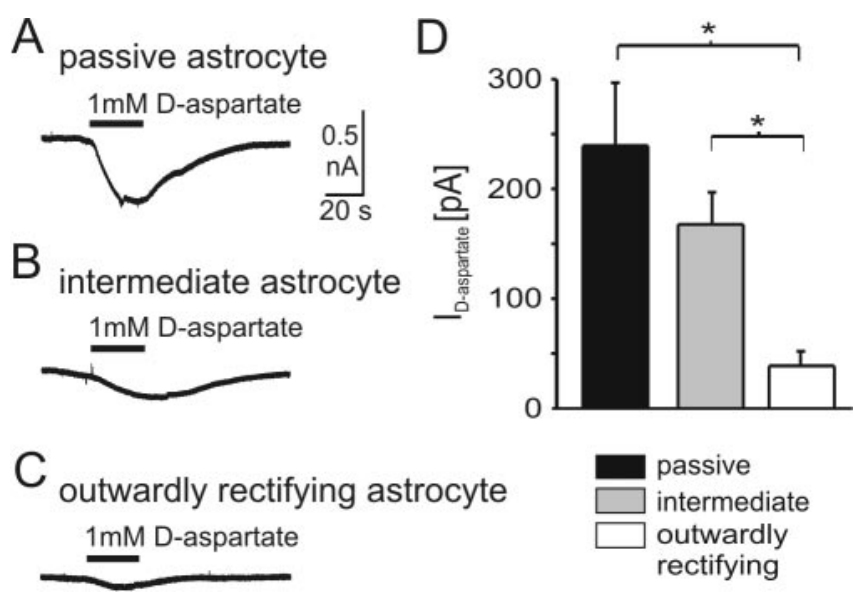

Figure 4. Transporter current expression in different types of astrocytes. A-C, Whole-cell voltage-clamp recordings of an intermediate astrocyte from the VRG during application of $1 \mathrm{~mm}$ D-aspartate. TTX ( $500 \mathrm{~nm}), \mathrm{CdCl}_{2}(100 \mu \mathrm{m})$, and CNQX $(50 \mu \mathrm{m})$ were added to prevent indirect effects by neighboring neurons. $D$, Amplitudes of current responses to $1 \mathrm{~mm}$ D-aspartate in the three types of astrocytes (data are given as mean $\pm \mathrm{SE}$ ).

and outwardly rectifying, voltage-gated potassium channel activation was found to be involved in the gene regulation of glial progenitor cells (Pende et al., 1994). AMPA receptor and outward rectifier expression could therefore serve as a marker for immature, undifferentiated, or progenitor cells [but see Matthias et al. (2003)].
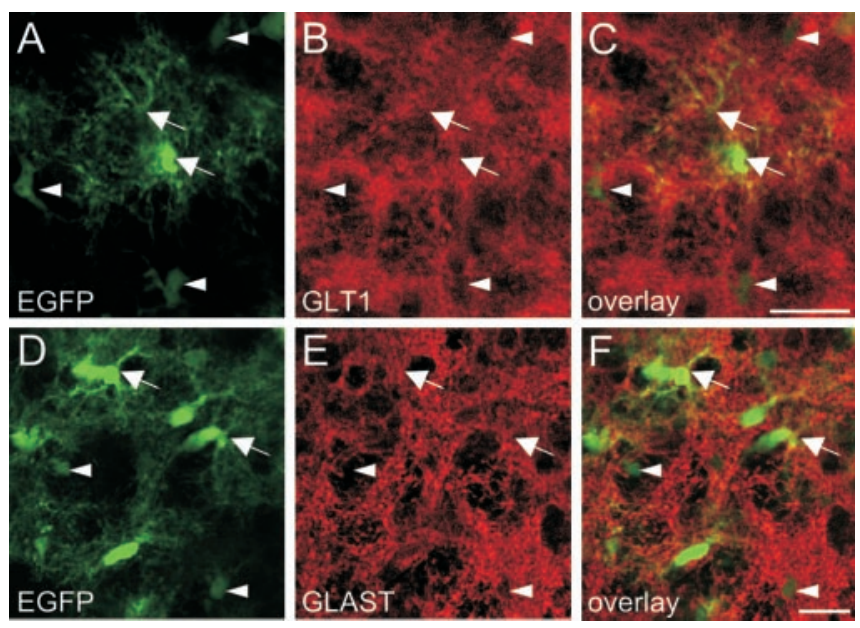

Figure 5. Immunohistochemistry for glial glutamate transporters. The expression of the glial glutamate transporters GLT-1 (A-C) and GLAST (D-F) is restricted to astrocytes with high expression levels of EGFP. A-C, Brainstem slices of P9 TgN(GFAP-EGFP) mice expressing EGFP in astrocytes $(A)$ were stained for GLT-1 $(B)$. As evident from the merged image $(C)$, astrocytes with high EGFP fluorescence (arrows) show overlapping GLT-1 labeling and EGFP fluorescence, whereas astrocytes with lower EGFP expression (arrowheads) do not show any expression of GLT-1. D-F, EGFP (D) and GLAST ( $E$ ) expression in astrocytes. Simultaneous expression ( $F$ ) of EGFP and GLAST was found in brightly fluorescent astrocytes (arrow); staining for GLAST was not detectable in astrocytes with low EGFP expression (arrowheads). Images are single optical sections of confocal microscopy. Scale bars: (in $C, F) A-F, 20 \mu \mathrm{m}$.

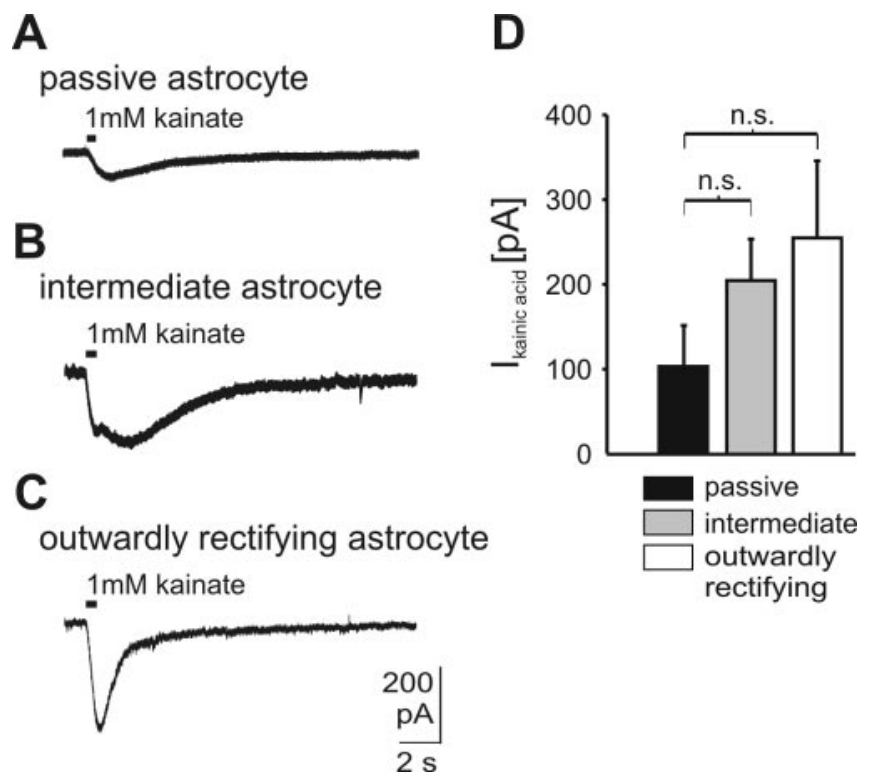

Figure 6. Effects of local application of kainate. All types of astrocytes responded to direct application of $1 \mathrm{~mm}$ kainate. $A-C$, Whole-cell voltage-clamp recordings of the three different types of astrocytes in the VRG during application of $1 \mathrm{~mm}$ kainate for 0.5 sec by pressure pulses; $500 \mathrm{~nm} \mathrm{TTX}$ and $100 \mu \mathrm{m} \mathrm{CdCl}_{2}$ were added to the bath to exclude indirect effects on recorded astrocytes. D, Amplitudes of current responses to $1 \mathrm{~mm}$ kainate in different types of astrocytes (n.S. = not significant; data are given as mean $\pm \mathrm{SE}$ ).

Conclusive with this hypothesis, kainate application (1 mM) elicited inward currents in outwardly rectifying astrocytes (242 \pm $93 \mathrm{pA} ; n=7$ ) (Fig. 6). The inward current in intermediate astrocytes was also comparable (192 $\pm 47 \mathrm{pA} ; n=12)$. In passive astrocytes, kainate also evoked an inward current, although it was slightly smaller (103 $\pm 41 \mathrm{pA} ; n=7)$; these differences, however, were not statistically significant. In all three types of astrocytes, kainate-induced inward currents were reversibly blocked by 
A

\section{passive astrocyte}

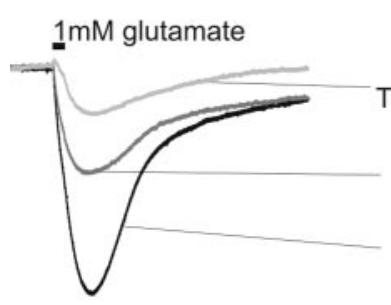

C

\section{passive astrocyte}

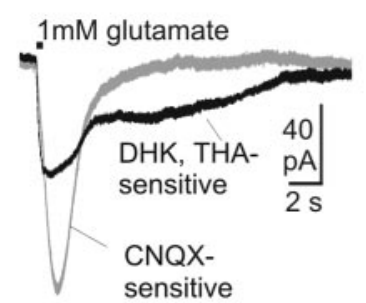

B outwardly rectifying astrocyte

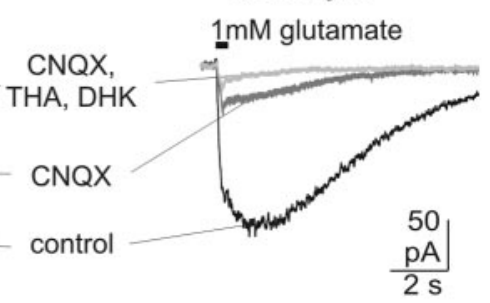

D

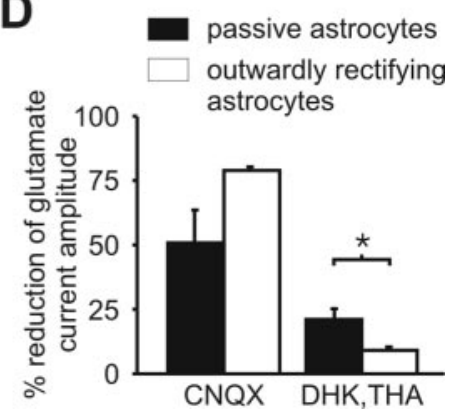

Figure 7. Glutamate-induced inward currents in passive and outwardly rectifying cells. Passive and outwardly rectifying astrocytes show a different sensitivity to glutamate receptor and transporter blockers. $A$, Current traces elicited by local application of $1 \mathrm{~mm}$ glutamate in a passive astrocyte under control conditions (500 nM TTX, $100 \mu \mathrm{M} \mathrm{CdCl}_{2}, 50 \mu \mathrm{M}$ AP5, $20 \mu \mathrm{M}$ bicuculline, $20 \mu \mathrm{m}$ strychnine) after blockade of AMPA receptors with $50 \mu \mathrm{m}$ CNQX and after additional blockade of glutamate transporters by $300 \mu \mathrm{m}$ DHK plus $50 \mu \mathrm{m}$ THA. B, Same experimental protocol in an outwardly rectifying astrocyte. C, Point-by-point subtraction of the recordings depicted in $A$ reveals different kinetics of the DHK-THA-sensitive and CNQX-sensitive currents. D, Reduction of glutamate current induced by receptor (CNQX) and transporter (THA + DHK) blockers in different types of astrocytes (data are given as mean $\pm \mathrm{SE}$ ).

CNQX (50 $\mu \mathrm{M}$, block to $24.9 \pm 5.3 \%$ of control; $n=6$; data not shown).

To substantiate the data obtained with agonists of glutamate transporters and AMPA receptors and to simultaneously visualize the time course of transporter and receptor currents, we recorded glutamate-induced currents in the presence of $500 \mathrm{nM}$ TTX, $100 \mu \mathrm{M} \mathrm{CdCl}, 100 \mu \mathrm{M}$ AP5, $10 \mu \mathrm{M}$ strychnine and $20 \mu \mathrm{M}$ bicuculline to block synaptic transmission and minimize indirect effects from neighboring neurons (Fig. 7). Glutamate (1 mM) elicited inward currents with amplitudes of $-112.0 \pm 54.2 \mathrm{pA}$ in passive astrocytes and $-90.2 \pm 35.6 \mathrm{pA}$ in outwardly rectifying astrocytes. In passive astrocytes only $50.8 \pm 13.9 \%$ of the glutamate-induced current was blocked by CNQX (50 $\mu \mathrm{M} ; n=$ $3)$. In outwardly rectifying astrocytes the effect of the glutamate receptor antagonist was larger: $78 \pm 1.3 \%$ of the glutamateinduced current was blocked by CNQX $(n=3)$. Additional blockade of glutamate uptake led to further reduction of the current. Coapplication of dihydrokainate (DHK; $300 \mu \mathrm{M})$ and $\beta$-threo-hydroxy-aspartate (THA; $50 \mu \mathrm{M}$ ) had a larger effect in passive astrocytes than in outwardly rectifying astrocytes. In passive astrocytes, the glutamate transporter antagonists DHK and THA caused an additional reduction of $20.0 \pm 2.6 \%(n=3)$ of initial glutamate current. This effect was significantly larger $(p<$ 0.05 ) than in outwardly rectifying astrocytes, in which only $9.0 \pm$ $1.3 \%(n=3)$ of the glutamate current was blocked by THA and DHK (Fig. 7A-C). Point-by-point subtraction revealed the faster kinetics of CNQX-sensitive currents (i.e., the AMPA receptor component) as compared with the THA-DHK-sensitive (i.e., transporter) currents (Fig. 7D).

Outwardly rectifying astrocytes represent a distinct astroglial cell population

The strong response to glutamate receptor agonist, almost no glutamate transporter current, a generally lower EGFP expression, the existence of A-type potassium currents, and lack of potassium inward currents in outwardly rectifying astrocytes suggested that they not only have different functions, but rather represent a distinct population or a different developmental state of astrocytes. To validate this assumption, we performed an immunohistochemical analysis of the expression of markers defining other differentiation stages; indeed, expression of the proteoglycan NG2, which identifies glial progenitor cells (Diers-Fenger et al., 2001), was found in astrocytes with a low EGFP expression (Fig. $8 B, F, K$ ). Astrocytes with high levels of EGFP did not show immunoreactivity for the NG2 antigen. NG2-positive weakly EGFP-fluorescent cells were found in postnatal day (P) 2-23 mice. At P2, 53\% of weakly fluorescent GFAP-positive cells were NG2 positive. At P23, the number of NG2-positive cells with low EGFP fluorescence was comparably high (63\%). To learn more about the apparently exclusive position of the outwardly rectifying astrocytes in the brainstem, we performed additional immunohistochemistry. Double staining against NG2 and GFAP at early postnatal ages (P2) revealed no GFAP staining in NG2positive cells of weak EGFP fluorescence within the ventral respiratory group, but there was a strong GFAP expression in bright cells (Fig. 8A-D). Additionally, we searched for the early neuronal marker TUC-4 (Quinn et al., 1999) to determine whether outwardly rectifying cells represent a neuronally differentiated cell population. In the ventral respiratory group (Fig. $8 E-I$ ) and in the hypoglossal nucleus (data not shown), we were unable to detect TUC-4-positive cells. Immunolabeling for TUC-4 detected in other brainstem nuclei, including the inferior olive, did not colocalize with either EGFP or NG2 (Fig. 8I-M).

\section{Discussion}

Stabilization of synaptic transmission by astrocytes

Our experiments in the ventrolateral medulla, incorporating the respiratory network, and the hypoglossal nucleus revealed a functional diversity of astrocytes. Three main populations of EGFP-labeled cells differed with respect to their expression of voltage-dependent currents, glutamate transporter, and surface membrane protein expression. Two of these populations (passive and intermediate astrocytes) share many morphological and functional characteristics. Apart from the expression of A-type potassium currents, there were no differences between passive and intermediate cells, suggesting that these cells represent a variation of a population of mature cell types that supports synaptic transmission. A large number of cells serve as stabilizing elements for synaptic transmission because they express functional glutamate transporters to clear neurotransmitter from the extracellular space. Additionally, these cells provide a sink for extracellular potassium by expressing a high resting membrane conductance for potassium. $\mathrm{K}^{+}$buffering and regulation of excitatory transmitter levels determine the ongoing rhythmic activity of respiratory neurons and, for that matter, any other type of nonrespiratory neurons that are intermingled with respiratory neurons.

\section{Putative role of outwardly rectifying astrocytes}

In contrast to the supportive function of astrocytes for synaptic function observed in passive and intermediate astrocytes, we identified outwardly rectifying astrocytes that cannot substan- 
tially contribute to $\mathrm{K}^{+}$buffering and transmitter uptake. High expression of A-type $\mathrm{K}^{+}$channels, ionotropic glutamate receptors from the AMPA type, and expression of the NG2 proteoglycan are characteristics of migrating and proliferating progenitor cells (Pende et al., 1994; Knutson et al., 1997; Wang et al., 2000; Diers-Fenger et al., 2001). We observed outwardly rectifying astrocytes in the ventral respiratory group and the hypoglossal nucleus of brainstem preparations, independent of their age. This is in line with recent data from hippocampus (Zhou and Kimelberg, 2001; Matthias et al., 2003). NG2-positive cells were found to be progenitors that could differentiate not only into astrocytes or oligodendrocytes (Diers-Fenger et al., 2001; Mallon et al., 2002) but also into neurons (Belachew et al., 2003). The lack of the early neuronal marker TUC-4 in NG2-expressing EGFPpositive cells suggests that outwardly rectifying astrocytes do not belong to a neuronally differentiated cell population, which favors an astroglial relationship of these cells. The lack of GFAP expression in NG2-positive weakly fluorescent cells, on one hand, and the fact that these cells appear independent of age, on the other hand, suggest that these cells are not a functional specialization of mature astrocytes but rather represent a distinct, specialized cell type. Correspondingly, astrocytes with almost identical physiological properties exist in the hippocampus and were termed "astron" (Matthias et al., 2003) to indicate an intermediate status of these cells between neurons and glia.

The data presented here still do not exclude the possibility that outwardly rectifying cells represent a cellular differentiation stage of the glial or neuronal cell lineage as suggested by Belachew et al. (2003). In either case, both options are potentially relevant to the postnatal development of the ventral respiratory group. This part of the brainstem, which is harboring a vital neuronal circuitry, has to be wired effectively to allow coordinated network interactions controlling breathing and other visceral functions immediately after birth. In this context, it is noteworthy that we did not detect TUC-4 labeling in the ventral respiratory group of the early postnatal brainstem (P2), indicating that neuronal network formation and principal wiring have been completed at birth. Reorganization and adaptation of the synaptic architecture, however, have to be tailored to functional requirements during postnatal development (for review, see Hilaire and Duron, 1999; Richter and Spyer, 2001). Especially during the first 2 postnatal weeks, there are ongoing changes in the density of glutamate receptors on respiratory neurons (Liu and Wong-Riley, 2002). Together with postnatal growth of the brainstem and the extension of dendrites, there is an obvious necessity for functional adaptation of synapses and remodeling of glial processes. Our study does not exclude the possibility of postnatal replacement of cells in the VRG; however, it favors astroglial plasticity rather than ongoing replacement of neurons.
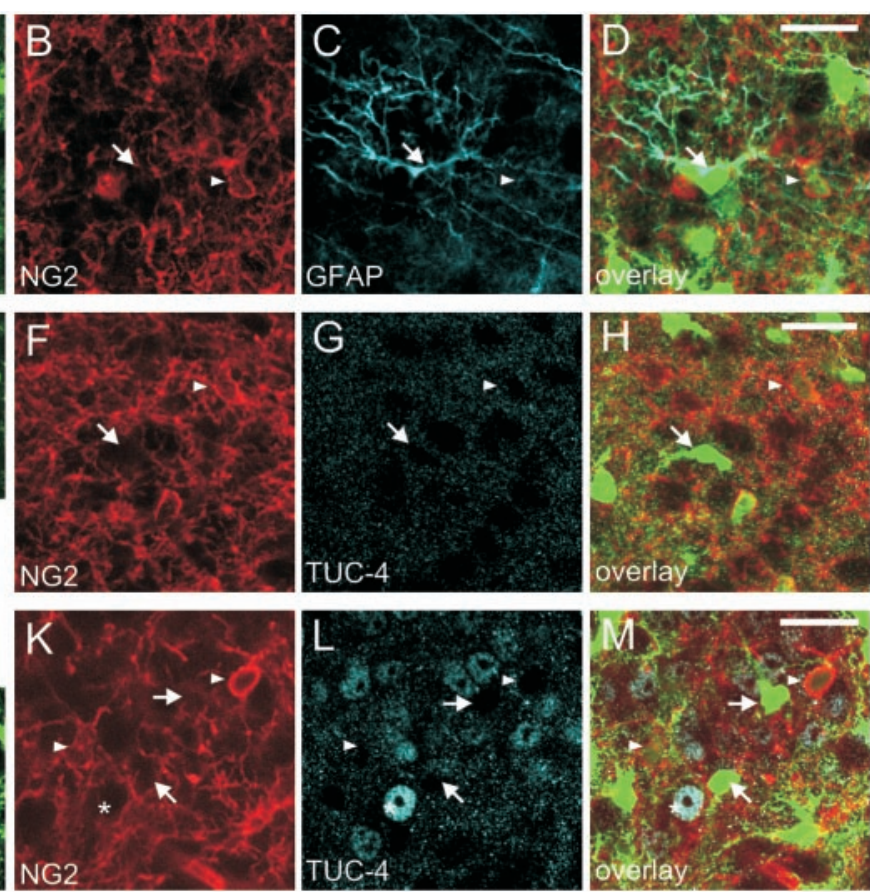

Figure 8. Immunohistochemistry for NG2, GFAP, and TUC-4. A-D, Double immunostaining in brainstem slices of P2 mice EGFP $(A)$, in astrocytes against the proteoglycan NG2 $(B)$, a glial progenitor marker, and GFAP $(C)$ in the ventra group $(I, G)$. Fluorescence staining for TUC-4 in the overview $/$ is depicted as inverted gray scale image. In the inferio ive, a region with TUC-4 expression ( $J-M$, asterisks), no overlap of NG2 and TUC-4 expression was found in astrocytes with low levels of EGFP fluorescence (arrowheads). Images represent single optical sections recorded by confocal microscopy. Scale bars: (in $D, H, M) A-H, J-M, 20 \mu \mathrm{m} ; \mathrm{I}, 200 \mu \mathrm{m}$.

\section{Functional relevance of coexpression of transporters and receptors}

In the present study, EGFP fluorescence was variable in the astrocytes of the respiratory network. Similar variability was found in hippocampus (Matthias et al., 2003) but not in Bergmann glia (Nolte et al., 2001). Matthias et al. (2003) found a one-to-one correlation of fluorescence intensity and cellular parameters, e.g., expression of glutamate receptors (low fluorescence) versus expression of glutamate transporter (high fluorescence). Additionally, they excluded coexpression of AMPA receptors and glutamate transporters in hippocampal astrocytes (Matthias et al., 2003). In contrast to hippocampal astrocytes, we observed coexpression of glutamate receptor and transporter currents in astrocytes with high EGFP fluorescence, both passive and intermediate astrocytes. These findings correspond to studies in Bergmann glia cells (Clark and Barbour, 1997), which interestingly also show high GFAP promoter-induced EGFP fluorescence (Nolte et al., 2001). This coexpression could well be of functional significance for the postnatal development of the respiratory network, because it has been shown in Bergmann glia that calciumpermeable AMPA receptors regulate the ensheathing of synapses by glial processes (Iino et al., 2001). Correspondingly, we have identified astrocytes in the respiratory network, e.g., passive and intermediate, that like Bergmann glia do combine properties for glutamate uptake and $\mathrm{K}^{+}$buffering, which can stabilize synaptic transmission, with receptive properties, e.g., AMPA receptors that potentially regulate synaptic plasticity. Additionally, other 
aspects of AMPA receptor-regulated adaptation of astroglial function have been discussed, e.g., glutamate transporter expression, potassium conductances, and gap-junctional coupling (Müller et al., 1992, 1996; Gegelashvili et al., 1996).

\section{Concluding remarks}

Our data show that astrocytes of the respiratory network represent a heterogeneous population of cells. Subsets of these cells, intermediate and passive astrocytes, fulfill the requirements necessary for stabilization of synaptic network activity. In addition, there is a distinct group of astroglial cells that could play a more dynamic role, allowing reorganization of the respiratory network during postnatal ontogeny and synaptic plasticity.

\section{References}

Belachew S, Chittajallu R, Aguirre AA, Yuan X, Kirby M, Anderson S, Gallo V (2003) Postnatal NG2 proteoglycan-expressing progenitor cells are intrinsically multipotent and generate functional neurons. J Cell Biol 161:169-186.

Bordey A, Sontheimer H (2000) Ion channel expression by astrocytes in situ: comparison of different CNS regions. Glia 30:27-38.

Brockhaus J, Ballanyi K, Smith JC, Richter DW (1993) Microenvironment of respiratory neurons in the in vitro brainstem-spinal cord of neonatal rats. J Physiol (Lond) 462:421-445.

Clark BA, Barbour B (1997) Currents evoked in Bergmann glial cells by parallel fibre stimulation in rat cerebellar slices. J Physiol (Lond) 502:335-350.

Del Negro CA, Morgado-Valle C, Feldman JL (2002) Respiratory rhythm: an emergent network property? Neuron 34:821-830.

Diers-Fenger M, Kirchhoff F, Kettenmann H, Levine JM, Trotter J (2001) AN2/NG2 protein-expressing glial progenitor cells in the murine CNS: isolation, differentiation, and association with radial glia. Glia 34:213-228.

Dong XW, Feldman JL (1999) Distinct subtypes of metabotropic glutamate receptors mediate differential actions on excitability of spinal respiratory motoneurons. J Neurosci 19:5173-5184.

Edwards FA, Konnerth A, Sakmann B, Takahashi T (1989) A thin slice preparation for patch clamp recordings from neurones of the mammalian central nervous system. Pflügers Arch 414:600-612.

Filippov V, Kronenberg G, Pivneva T, Reuter K, Steiner B, Wang LP, Yamaguchi M, Kettenmann H, Kempermann G (2003) Subpopulation of nestin-expressing progenitor cells in the adult murine hippocampus shows electrophysiological and morphological characteristics of astrocytes. Mol Cell Neurosci 23:373-382.

Gegelashvili G, Civenni G, Racagni G, Danbolt NC, Schousboe I, Schousboe A (1996) Glutamate receptor agonists up-regulate glutamate transporter GLAST in astrocytes. NeuroReport 8:261-265.

Greer JJ, Smith JC, Feldman JL (1991) Role of excitatory amino acids in the generation and transmission of respiratory drive in neonatal rat. J Physiol (Lond) 437:727-749.

Hilaire G, Duron B (1999) Maturation of the mammalian respiratory system. Physiol Rev 79:325-360.

Hülsmann S, Oku Y, Zhang W, Richter DW (2000a) Metabotropic glutamate receptors and blockade of glial Krebs cycle depress glycinergic synaptic currents of mouse hypoglossal motoneurons. Eur J Neurosci 12:239-246.

Hülsmann S, Oku Y, Zhang W, Richter DW (2000b) Metabolic coupling between glia and neurons is necessary for maintaining respiratory activity in transverse medullary slices of neonatal mouse. Eur J Neurosci $12: 856-862$.

Iino M, Goto K, Kakegawa W, Okado H, Sudo M, Ishiuchi S, Miwa A, Takayasu Y, Saito I, Tsuzuki K, Ozawa S (2001) Glia-synapse interaction through $\mathrm{Ca}^{2+}$-permeable AMPA receptors in Bergmann glia. Science 292:926-929.

Jabs R, Kirchhoff F, Kettenmann H, Steinhauser C (1994) Kainate activates $\mathrm{Ca}\left({ }^{2+}\right)$-permeable glutamate receptors and blocks voltage-gated $\mathrm{K}^{+}$ currents in glial cells of mouse hippocampal slices. Pflügers Arch 426:310-319.

Knutson P, Ghiani CA, Zhou JM, Gallo V, McBain CJ (1997) $\mathrm{K}^{+}$channel expression and cell proliferation are regulated by intracellular sodium and membrane depolarization in oligodendrocyte progenitor cells. J Neurosci 17:2669-2682.

Koshiya N, Smith JC (1999) Neuronal pacemaker for breathing visualized in vitro. Nature 400:360-363.

Kullmann DM, Asztely F (1998) Extrasynaptic glutamate spillover in the hippocampus: evidence and implications. Trends Neurosci 21:8-14.

Liu Q, Wong-Riley MT (2002) Postnatal expression of neurotransmitters, receptors, and cytochrome oxidase in the rat pre-Botzinger complex. J Appl Physiol 92:923-934.

Mallon BS, Shick HE, Kidd GJ, Macklin WB (2002) Proteolipid promoter activity distinguishes two populations of NG2-positive cells throughout neonatal cortical development. J Neurosci 22:876-885.

Matthias K, Kirchhoff F, Seifert G, Huttmann K, Matyash M, Kettenmann H, Steinhauser C (2003) Segregated expression of AMPA-type glutamate receptors and glutamate transporters defines distinct astrocyte populations in the mouse hippocampus. J Neurosci 23:1750-1758.

Mennerick S, Shen W, Xu W, Benz A, Tanaka K, Shimamoto K, Isenberg KE, Krause JE, Zorumski CF (1999) Substrate turnover by transporters curtails synaptic glutamate transients. J Neurosci 19:9242-9251.

Müller T, Möller T, Berger T, Schnitzer J, Kettenmann H (1992) Calcium entry through kainate receptors and resulting potassium-channel blockade in Bergmann glial cells. Science 256:1563-1566.

Müller T, Fritschy JM, Grosche J, Pratt GD, Mohler H, Kettenmann H (1994) Developmental regulation of voltage-gated $\mathrm{K}^{+}$channel and $\mathrm{GABA}_{\mathrm{A}}$ receptor expression in Bergmann glial cells. J Neurosci 14:2503-2514.

Müller T, Möller T, Neuhaus J, Kettenmann H (1996) Electrical coupling among Bergmann glial cells and its modulation by glutamate receptor activation. Glia 17:274-284.

Nolte C, Matyash M, Pivneva T, Schipke CG, Ohlemeyer C, Hanisch UK, Kirchhoff F, Kettenmann H (2001) GFAP promoter-controlled EGFPexpressing transgenic mice: a tool to visualize astrocytes and astrogliosis in living brain tissue. Glia 33:72-86.

Pende M, Holtzclaw LA, Curtis JL, Russell JT, Gallo V (1994) Glutamate regulates intracellular calcium and gene expression in oligodendrocyte progenitors through the activation of DL-alpha-amino-3-hydroxy-5methyl-4-isoxazolepropionic acid receptors. Proc Natl Acad Sci USA 91:3215-3219.

Quinn CC, Gray GE, Hockfield S (1999) A family of proteins implicated in axon guidance and outgrowth. J Neurobiol 41:158-164.

Richter DW, Spyer KM (2001) Studying rhythmogenesis of breathing: comparison of in vivo and in vitro models. Trends Neurosci 24:464-472.

Richter DW, Camerer H, Sonnhof U (1978) Changes in extracellular potassium during the spontaneous activity of medullary respiratory neurones. Pflügers Arch 376:139-149.

Robinson D, Ellenberger H (1997) Distribution of N-methyl-D-aspartate and non- $N$-methyl-D-aspartate glutamate receptor subunits on respiratory motor and premotor neurons in the rat. J Comp Neurol 389:94-116.

Rusakov DA, Kullmann DM (1998) Extrasynaptic glutamate diffusion in the hippocampus: ultrastructural constraints, uptake, and receptor activation. J Neurosci 18:3158-3170.

Rybak IA, Shevtsova NA, St. John WM, Paton JF, Pierrefiche O (2003) Endogenous rhythm generation in the pre-Botzinger complex and ionic currents: modeling and in vitro studies. Eur J Neurosci 18:239-257.

Smith JC, Ellenberger HH, Ballanyi K, Richter DW, Feldman JL (1991) PreBotzinger complex: a brainstem region that may generate respiratory rhythm in mammals. Science 254:726-729.

Stewart RR, Zigova T, Luskin MB (1999) Potassium currents in precursor cells isolated from the anterior subventricular zone of the neonatal rat forebrain. J Neurophysiol 81:95-102.

Wang JY, Wang J, Golovina VA, Li L, Platoshyn O, Yuan JX (2000) Role of $\mathrm{K}(+)$ channel expression in polyamine-dependent intestinal epithelial cell migration. Am J Physiol Cell Physiol 278:C303-314.

Zhou M, Kimelberg HK (2001) Freshly isolated hippocampal CA1 astrocytes comprise two populations differing in glutamate transporter and AMPA receptor expression. J Neurosci 21:7901-7908. 